\title{
Clinical Pharmacy Interventions in Pediatric Intensive Care Unit: A Review
}

\section{Annette Mariam Mathew and Juny Sebastian*}

Department of Pharmacy Practice, JSS College of Pharmacy, JSS Academy of Higher

Education and Research, Mysuru, India

*Corresponding Author: Juny Sebastian, Department of Pharmacy Practice, JSS College of Pharmacy, JSS Academy of Higher Education and Research, Mysuru, India.
Received: May 14, 2021

Published: June 29, 2021

(C) All rights are reserved by Annette Mariam Mathew and Juny Sebastian.

\begin{abstract}
Background: Clinical pharmacy is considered as the essential part of healthcare team in a hospital setting providing pharmaceutical care services to ensure the rational drug use. The outcome of clinical pharmacy intervention is the improvement of treatment effectiveness by providing alternative suggestions on the drug related problems and medication errors.

Methods: Extensive literature search was performed using various search engines. The identified articles were screened and segregated based on the inclusion criteria of studies on clinical pharmacy intervention in pediatric intensive care unit to prepare the review article.

Results: Clinical pharmacist's involvement ensures in assessing, recommending and prevention of any event involving drug therapy that potentially interfere with health outcomes. Various studies shows that dose alteration and pharmacokinetic recommendation are the most common interventions made by clinical pharmacists. There are certain factors that can induce the occurrence of drug related problems (DRPs) including polypharmacy, disease comorbidities, and long hospitalization and transferred admissions. Studies found the incidence rate of 5.5 interventions per patient in pediatric intensive care units (PICU) that has significant impact on the health outcome. Specific consideration should be given to special population including pediatrics who are more likely to develop DRPs as pharmacodynamics and kinetic behaviors of drugs in this population differfrom adults.

Conclusion: The identified contributing factors for DRPs in PICU are polypharmacy, long duration of hospital stay and comorbidities. As a part of the health care team, clinical pharmacist can review the treatment charts on daily basis to identify and suggest appropriate recommendations on identification of any DRPs or medication errors. These interventions can significantly improve the clinical and economical outcomes of patients admitted in PICUs.
\end{abstract}

Keywords: Clinical Pharmacy Intervention; Drug Related Problems; Medication Error; Pediatrics

\section{Introduction}

American academy of pediatrics defines pediatrics as a group of medical science concerned with physical, mental and social health of children from young adulthood. Pediatric population is among the special population where the drug treatment provided to them should be safe and effective. Due to lack of specific research on pediatrics pharmacotherapy and high usage of off label medica- tions, the children are at high risk of medication errors [1]. Off label prescriptions are very common in pediatrics, so it is very essential to rationalize the pediatrics pharmacotherapy. The rationalized use of medication in this population must be carried out by a team which is proficient with the medication therapy. American College of Clinical Pharmacy defines clinical pharmacy as a unit of health science including pharmacists who provide pharmaceutical care to 
the patients with optimization of medication therapy and improvement of health, wellness and disease prevention. They play an important role in health care by accompanying the multidisciplinary team on providing better pharmacotherapy and patient care [2]. A report of WorldHealth Organization in 2005 shows three times more medical errors in pediatrics than in adults [3]. Inclusion of clinical pharmacist in the medical team can improve drug selection and information on dose maintenance concerned with critical care patients $[1,4]$.

\section{Methods}

The review was performed from October 2020 to March 2021.

\section{Search strategies}

The review was prepared after an extensive literature search using the following key words- clinical pharmacy interventions, drug related problems (DRP) in Pediatric Intensive Care Unit (PICU), medication error, and content validity index, interventions at PICU, role of pharmacistin PICU using search engines such as Google scholar, Science Direct, PubMed/Medline and textbooks of Clinical pharmacy [1-19].

\section{Selection criteria}

Full text articles which were accessible were included in the review process as these articles defines the information on clinical pharmacy interventions and pharmaco-therapeutic treatments to patients in pediatric intensive care unit. This included research articles on clinical pharmacy interventions in pediatric intensive care unit. Research articles that did not focus on pharmacy activities in PICU and the inaccessible articles were excluded from the study.

\section{Review process}

Researchers independently reviewed the selected articles and screened the title abstract, keywords and followed by full text articles. Characteristics of studies considered to include in this review were study design, study population, study duration, study site. Review also considered role of clinical pharmacist in managing DRPs in PICU settings. Piloting of literature screening and data extraction was done to standardize the review and data extraction process.

\section{Results}

A total of 20 articles were included for the preparing the review article as per the article selection criteria. Flow diagram of study selection is presented in figure 1.

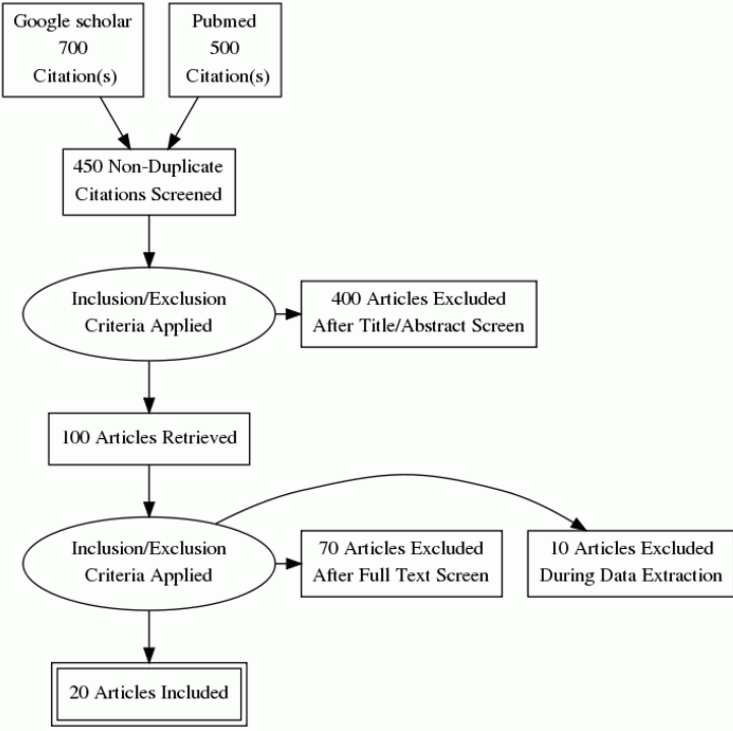

Figure 1: Flow diagram of study selection.

Clinical pharmacy interventions describe the activity of the pharmacist relating to patient care together with identification, correction and prevention of DRPs. With the assessment of medication prescription and dispensing, higher safety as well as monetary advantages will be achieved together with shorter length of stay, reduced DRPs and mortality [5]. Recommendation can be provided by the clinical pharmacy for the resolution of the identified drug related problem. These interventions could also be performed with varied clinical pharmacyactivities together with medication history interview, medical chart review and therapeutic drug monitoring. Various studies show that medication error is the commonest intervention by a pharmacist within the pediatric setting, among that dose calculation errors are most frequent [6].

Appropriate communication with the multidisciplinary team, with parents or bystanders and correct review of medical chart, would help prevent occurrence of medication errors to a greater extend. Therefore inclusion of a pharmacist in a health care team is very necessary and in some of the developed countries like United States of America determines the pharmacist as an all time member in the medical team in the hospitals [2]. Impact of clinical pharmacy intervention in adult inpatient settings had been reported fromvaried studies, nevertheless the impact of pharmacy intervention in pediatric population has not been documented. 
Clinical pharmacy interventions can be categorized into different groups including Dosing recommendation, antibiotic recommendation, and pharmacokinetic recommendation, changes in routes of administration, drug interactions, and renal dose adjustment recommendations [8].

Dosing recommendations include change and corrections in the dose that is important inchildren as it to be done based on the body weight. Various studies shows that dosing recommendation is the most commonly occurred pharmacy intervention in PICU. Overdosing and under dosing is observed that include dose adjustment of supplemental medication and IV administrations.

Pharmacokinetic recommendation include opting better therapeutic choice based on the determination of drug levels.

Addition, substitution and discontinuation of drugs as a better therapeutic choice has beenobserved, antimicrobial medications are been mostly included in this section.

Antibiotic recommendation include suggesting particular antibiotic regimen for treatment according to the reported culture sensitivity and antibiotic policy and guidelines are altering the dose and frequencies based on the spectrum of activity and renal functions where additional monitoring is required.

Change in the route of administration, from intravenous to oral route as it's more preferred to reduce the cost and prevent infections.

Alteration of drug therapy due to drug- drug interaction, drugfood interactions includes tapering the particular drug or choosing another drug without any drug interaction and maintaining the administration time gap by checking the pharmacokinetic properties of the drug regimen. Patient counselling on intake of food and diet is required if any drug-food interaction been found.

Also, Clinical Pharmacist can help the health care team to identify, report and address Drug Related Problems and medication errors by appropriate interventions [19].

Dose alterations and pharmacokinetic recommendations are the most common interventions observed [7]. The categorized interventions can be again grouped based on the severity of interventions and impact on patient outcome as minor, moderate and life-threatening. A 11 year study done in PICU observed that the determined intervention had a moderate impact on patient outcome, which includes dose adjustment, failure to receive medication and electrolyte imbalance [8]. Thus better the outcome of an intervention in patient care directly denotes the importance of a clinical pharmacy service in pediatric intensive care setting.

\section{Drug related problems}

Drug related problem is defined as an event or circumstance involving drug therapy that actually or potentially interferes with health outcomes [9]. The pediatric population is of more risk to be affected by DRPs as the pharmacokinetic and pharmacodynamics states are different from adults [10]. Helper and strand classified the drug related problems into eight categories which is described in figure 2:

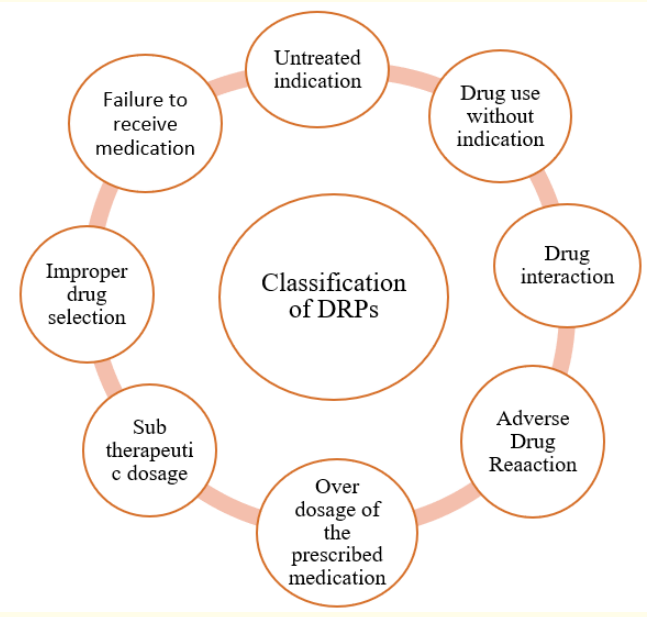

Figure 2: Classification of drug related problem based on helper and strand classification.

- Untreated indication: Where patient is not getting treated with the required medication for the affected medical problem, on reviewing its important to notice whether the indication is an unrecognized ADR. Some of the untreated indications may not be clear as it's not presenting any signs and symptoms.

- Improper drug selection: Selecting wrong medication that's not related to the medical problem, so it's important to ensure that appropriate drug has been selected for the treatment. The selection of drug for therapy should be thoroughly based on considering the patients clinical situations. Also ensure that the prescribed drugs are able to achieve the therapeutic objectives. 
- Sub therapeutic dosage: Here the required dose of the medication is not received by thepatient, as a result a sub therapeutic effect is induced. The dose and dosing regimen should be individualized based on the patients' medical condition. Clinical pharmacokinetic parameters can be monitored like the serum concentration, therapeutic drug monitoring which is a useful aid in determining the dose frequency.

- $\quad$ Failure to receive medication: Patient gets fail to receive a medication and getting a medical problem. This occurs due to different factors including non-adherence, poor administration technique, missed doses due to medication errors, substandard drugs, non- availability of the prescribed drugs.

- Over dosage of the prescribed medication: Over dosage may occur because of several reasons including calculation error, as dose is not calculated based on body weight and the recommended guidelines and policies, if a patient takes a drug for a long time without following the particular prescription order, self-medications without the prescriptions and importantly if the same generic drug has been prescribed twice under different brand names. Lack of education and carelessness can lead to this conditions. So it's important to monitor the individual doses and total daily dose.

- Drug interactions including drug-drug interactions, drugfood interactions: Drug interactions should be monitored and its decision making on alteration of drug therapy should be based on the severity of the interactions. Drug interactions might be either potential or actual drug interactions. Potential drug interaction occur when two drugs known to interact are concurrently prescribed, regardless of whether adverse event occur. In actual drug interaction, clinically meaningful alteration of the effect of a drug occursas a result of co-administration of another drug. Pharmacist have to consider a resolution for the reported interactions involving the essential medications which can include the change in frequency.

- $\quad$ Adverse drug reactions: On prescribing a drug pharmacist need to ensure about the patients past medical history and is the patient is allergic to any medications. Additionally it's important to assess the patient for the presence of any new symptom on administration of drugs. All patients, especially those who are most susceptible to develop an ADR, should be monitored on daily basis. The identified ADRs can be assessed for its causality using Naranjo's algorithm, preventability using Schumock and Thornton Scale, severity using Hartwig and
Siegel Severity Assessment Scale and seriousness by FDA Criteria. ADR due to Drug interactions are assessed using Drug interaction probability Scale.

- Drug use without indication: Patient receiving medication for an invalid indication. Indeed it need a special monitoring, as the indication for which a drug is used may not be immediately accurate. Certain drugs have multiple indications. Pharmacist should ascertain the intervention only with thorough knowledge and practical experience $[11,17]$.

Sub therapeutic dosing, over dosing and drug interactions are the common drug related problems that can occur in pediatric settings. Considering the pediatric patients in critical care units, thereis more chance of drug interactions and adverse drug reactions as the patients will be on multiple therapy and certain high risk medications which requires close monitoring, this marks the necessity of a clinical pharmacist in pediatric intensive care units.

\section{Predictors of drug related problems}

Predictors are the factors which is associated with the occurrence of drug related problems. The drug related problems arise due to different reasons. The factors contributing to the occurrence ofdrug related problems include missing information, half knowledge of the patients, polypharmacy, disease conditions with certain comorbidities, renal impairment, language issues, long hospital stay, transferred admissions, additional physician office visits andadditional prescriptions $[6,12]$.

A study conducted in Hong Kong at a pediatric ward shows that long hospitalization,additional physician office visit and additional prescriptions were some of the consequences associated with DRPs. The study states that patients with multiple medications and severe infectious diseases are more likely experience the drug related problems ${ }^{12}$. In certain other investigations transferred admissions were also observed as the potential risk factor of DRPs in PICUs [6]. Non adherence is an expected predictor of DRPs especially among the pediatric population which is due to certain factors like family situations such as poor parenteral care, limited education, limited understanding and developmental abilities of child. Certain regimen factors including complexity, cost, type of regimen and taste of the medication contributes to the non-compliance.

\section{Medication errors}

Medication error is any preventable event that may cause or lead to inappropriate medication use or patient harm while the 
medication is in the control of the health care professional, patient, or consumer. Medication error occur due to human mistakes or any system damages. This can happen from prescription to medication administration. There are several factors that contribute to the occurrence of medication error which includes calculation error, sound alike and look alikemedications, emergency situations, duty shift, new staff, patient transfer, stress, and storage issues. A former study suggest that medication errors are more harmful in children than in adults [5]. Due to emergency situations, use of high alert medications, duty shifts and workload of the staffs there is high chance of occurrence of medication error in inpatient settings. Medication errors are highly harmful to the children admitted in pediatric intensive care units as they get treated with multiple medications [13].

A previous study conducted in Netherlands on evaluation of medication error in pre intervention and post intervention state, suggest that implementation of a clinical pharmacist with an ordered medication review results in a significant reduction in medication error [1].

The categories of medication error based on the National Coordinating Council on Medication Error and Reporting Prevention (NCCMERP) is shown in table 1.

\begin{tabular}{|c|c|}
\hline Categories & Description \\
\hline \multicolumn{2}{|l|}{ No error } \\
\hline A & $\begin{array}{l}\text { Circumstances or events that have the capac- } \\
\text { ity to cause error }\end{array}$ \\
\hline \multicolumn{2}{|l|}{ Error, no harm } \\
\hline B & $\begin{array}{c}\text { An error occurred, but the medication did not } \\
\text { reach the patient }\end{array}$ \\
\hline $\mathrm{C}$ & $\begin{array}{l}\text { An error occurred that reached the patient but } \\
\text { did not cause patient harm }\end{array}$ \\
\hline $\mathrm{D}$ & $\begin{array}{c}\text { An error occurred that resulted in the need for } \\
\text { increased patient monitoring but no patient } \\
\text { harm }\end{array}$ \\
\hline \multicolumn{2}{|l|}{ Error, harm } \\
\hline E & $\begin{array}{c}\text { An error occurred that resulted in the need for } \\
\text { treatment or intervention and caused tempo- } \\
\text { rary patient harm }\end{array}$ \\
\hline $\mathrm{F}$ & $\begin{array}{l}\text { An error occurred that resulted in initial or } \\
\text { prolonged hospitalization and caused tempo- } \\
\text { rary patient harm }\end{array}$ \\
\hline G & $\begin{array}{l}\text { An error occurred that resulted in permanent } \\
\text { patient harm }\end{array}$ \\
\hline \multicolumn{2}{|l|}{ Error, death } \\
\hline I & $\begin{array}{l}\text { An error occurred that resulted in patient } \\
\text { death }\end{array}$ \\
\hline
\end{tabular}

Table 1: NCC MERP definition of a medication error and risk assessment.
A prospective cohort study conducted by Bates., et al. has observed that, preventable ADRS and ADEs were commonly occurred among his study population. He concludes that most of the medication errors were occurred at the prescribing and administrating stage [14].

\section{Factors affecting occurrence of medication errors}

A comparison study among 7 countries on determinants of patient reported medication error, shows the risk factors associated with medication error including poor coordination in care, and expense related issues, increasedhospital length of stay. WHO in 2016 states the factors influencing medication errors as:

- $\quad$ Lack of knowledge, workload and physical and emotional status of the healthcareprofessionals.

- $\quad$ Error in naming, labeling and packing of medicines.

- Complexity of clinical and polypharmacy

- $\quad$ Repetition in prescription

- $\quad$ Limited communication and coordination in secondary care.

In a study conducted in UK, they interviewed the medical team and characterized the factors associated with medication errors into 5 groups including individual factors, organizationalfactors, risk related factors, team related factors, and work related factors. With individualanalyzation of these different factors and reviewing each of the error groups by a pharmacist can reduce the medication related problems and can improve the role of clinical pharmacist in medication reviews. Also a detailed review of the medical record, detection of the medical errors and discussing it with the physician can reduce the occurrence of errors and can improve impact of clinical pharmacist interventions [13].

Medications involved in clinical pharmacy intervention in PICU

The class of medications or drugs which are majorly involved in drug related problems can be determined by the categorization of the drugs based on the class it belongs to. Certain studies shows that antibiotics medications are the ones mostly associated with DRPs, analgesics, gastrointestinal agents, and anti-anemics are the other drug classes commonly associated with DRPs. The interventions on antibiotics commonly include increasing the dose of the medication to reach the spectrum activity [7]. Previously conducted studies have observed that among the antibiotic medications vancomycin is commonly associated to DRPs $(36.4 \%, 6.8 \%)[7,15]$. 


\section{Discussion}

This review was done to know about the clinical pharmacist activities in pediatric intensive care unit. Eight months to elevenyear studies had been conducted on describing the impact of clinical pharmacy interventions within the pediatric inpatient settings. These studies show the various categorization of interventions performed and also the most typically occurred ones that integrates the cost and length of stay in inpatient settings $[7,8]$. During the study period thepresence of a clinical pharmacist in PICU reduced the incidence of interventions by $10 \%$. It also shows that inclusion of a unit based pharmacist had prevented $94 \%$ of potential adverse drug events. Here with improved working hours of pharmacist prevents $21.1 \%$ of medication errors. From the overall interventions performed the proportion of drug dosing interventions faded from $65 \%$ to 42 in 11 years.

The leading objective of clinical pharmacist is the assessment of drug related problems and intervening it with the medical team to reduce the risk and for better patient outcome onpharmacotherapy. Considering pediatric intensive care patients it's very essential to assess the medication therapy and the patient benefit voiding the associated risks. On reviewing the interventions obtained in an 11 year study shows that most of the identified interventions were having a moderate impact on the better patient outcome. A single centered randomized controlled trial by Zhang., et al. shows $96.8 \%$ of clinical acceptance, where the study conducted in Cot D'ivore shows $67.3 \%$ of acceptance of intervention in which $16 \%$ have very significant clinical impact.

\begin{tabular}{|c|c|c|c|}
\hline Studies & $\begin{array}{c}\text { Common } \\
\text { intervention }\end{array}$ & Drugs involved & $\begin{array}{c}\text { \% of } \\
\text { acceptance }\end{array}$ \\
\hline $\begin{array}{c}\text { La } \\
\text { Rochelle., } \\
\text { et al. }\end{array}$ & $\begin{array}{c}\text { Drug Dosing } \\
\text { Pharmacoki- } \\
\text { netics }\end{array}$ & $\begin{array}{c}\text { Antibiotics } \\
\text { (vancomycine) } \\
\text { Sedatives, analgesics } \\
\text { GI drugs }\end{array}$ & 98 \\
\hline $\begin{array}{c}\text { Okumura., } \\
\text { et al. }\end{array}$ & $\begin{array}{c}\text { Improve drug } \\
\text { therapy } \\
\text { Dose } \\
\text { alteration }\end{array}$ & $\begin{array}{c}\text { Antibiotics((merope } \\
\text { nam,vancomycine) }\end{array}$ & 74 \\
\hline $\begin{array}{c}\text { Aborgoua., } \\
\text { et al. }\end{array}$ & $\begin{array}{c}\text { Dose } \\
\text { adjustment } \\
\text { therapeutic } \\
\text { choice }\end{array}$ & $\begin{array}{c}\text { Anti-anemics ,Anti- } \\
\text { biotics }\end{array}$ & 94.8 \\
\hline
\end{tabular}

Table 2: Comparison on studies and the common pharmacy interventions and acceptance.
Better patient outcome from the interventions need to be considered on determining the impact of clinical pharmacist. A cross sectional study conducted in Brazil had found that $74 \%$ of all PICU variations in length of stay were associated with drug related problems. Intervening, correcting and monitoring of these drug related problems can reduce the length of stay as well as the hospital expenditure. The higher the rate of acceptance and better the patient benefits, higher the impact of clinical pharmacy intervention within medical team. From the review of variant studies, it's clear that patient outcome with intervention can directly improve the role of clinical pharmacist compared to the clinical acceptance. It improves the role of clinical pharmacist in pharmacotherapy in pediatric inpatient setting $[16,20]$.

\section{Conclusion}

Clinical pharmacy intervention can implement a safe and efficacious treatment strategy by reducing the occurrence of drug related problems. The drug related problems can be minimized by identifying the predictors and monitoring it. From review of various research articles it's clear that clinical pharmacist are the essential part of multidisciplinary healthcare team who have a great role in assessing and recommending the best treatment options thus providing better patientcare.

\section{Acknowledgement}

The authors would like to thank the administrators and staff of Department of Pharmacy PracticeJSS College of Pharmacy, JSS Academy of Higher Education and Research, Mysore for their support and encouragement in completing this article.

\section{Funding}

No funding.

\section{Conflict of Interest}

No conflict.

\section{Bibliography}

1. Dersch-Mills D. "Assessment Considerations in Pediatric Patients". Inpatient Assessment in Clinical Pharmacy 2019 Springer, Cham 387-401.

2. Maaskant JM., et al. "Medication audit and feedback by a clinical pharmacist decrease medication errors at the PICU: An interrupted time series analysis". Health Science Reports 1.3 (2018): e23. 
3. Conroy S., et al. "Survey of unlicensed and off label drug use in paediatric wards in European countries". Bmj 320.7227 (2000): 79-82.

4. Gorman RL., et al. "Prevention of medication errors in the pediatric inpatient setting". Pediatrics 112.2 (2003): 431.

5. Kaushal R., et al. "Medication errors and adverse drug events in pediatric inpatients". JAMA 285 (2001): 2114-2120.

6. Ghaleb MA., et al. "The incidence and nature of prescribing and medication administration errors in paediatric inpatients". Archives of Disease in Childhood 95.2 (2010): 113-118.

7. LaRochelle JM., et al. "Clinical pharmacy faculty interventions in a pediatric intensive care unit: an eight-month review". The Journal of Pediatric Pharmacology and Therapeutics 17.3 (2012): 263-269.

8. Tripathi S., et al. "Impact of clinical pharmacist on the pediatric intensive care practice: an 11-year tertiary center experience". The Journal of Pediatric Pharmacology and Therapeutics 20.4 (2015): 290-298.

9. Daniel L., et al. "An Investigation on Drug Related Problems in Pediatrics of a Tertiary Care, Private, Teaching Hospital at Coimbatore". Journal of Clinical Case Reports and Trials 1 (2018): 1-7.

10. Kearns GL., et al. "Developmental pharmacology-drug disposition, action, and therapy in infants and children". New England Journal of Medicine 349.12 (2003): 1157-1167.

11. American Society of Hospital Pharmacists. "ASHP statement on pharmaceutical care". American Journal of Health-System Pharmacy 50 (1993): 1720-1723.

12. Rashed AN., et al. "Epidemiology and potential risk factors of drug-related problems in Hong Kong paediatric wards". British Journal of Clinical Pharmacology 77.5 (2014): 873-879.

13. Sutherland A., et al. "Exploring the human factors of prescribing errors in paediatric intensive care units". Archives of Disease in Childhood 104.6 (2019): 588-595.
14. Bates DW., et al. "Incidence and preventability of adverse drug events in hospitalized adults". Journal of General Internal Medicine 8.6 (1993): 289-294.

15. Okumura LM., et al. "Relation between safe use of medicines and clinical pharmacy services at pediatric intensive care units". Revista Paulista de Pediatria 34.4 (2016): 397-402.

16. Abrogoua DP., et al. "Assessment of a clinical pharmacy activity in a pediatric inpatient department in Cote d'Ivoire". Journal of Basic and Clinical Pharmacy 8.1 (2016): 15.

17. Parthasarathi G., et al. "A Textbook of Clinical Pharmacy Practice Essential Concept and Skill". (1 st edn) Oriental Longman Private Limited 193-245.

18. Viana SD., et al. "Interventions of the clinical pharmacist in an Intermediate Care Unit for elderly patients". Einstein (São Paulo) 15.3 (2017): 283-288.

19. Jafarian K., et al. "The responsibility of clinical pharmacists for the safety of medication use in hospitalized children: A Middle Eastern experience". Journal of Research in Pharmacy Practice 8.2 (2019): 83.

20. Malfara M., et al. "Impact of the clinical pharmacist interventions on prevention of pharmacotherapy related problems in the paediatric intensive care unit". International Journal of Clinical Pharmacy 40.3 (2018): 513-519.

\section{Volume 5 Issue 7 August 2021 (C) All rights are reserved by Annette Mariam Mathew and Juny Sebastian.}

